\title{
Analysis of stereotyped voluntary movements at the elbow in patients with Parkinson's disease ${ }^{1}$
}

\author{
M. H A L L E T T ${ }^{2}$, B. T. SH A H A N I, A N D R.R. YOU NG ${ }^{3}$ \\ From the Department of Neurology and the Laboratory of Clinical Neurophysiology, \\ Massachusetts General Hospital and Harvard Medical School, Boston, Massachusetts, USA
}

S U M M A R Y Patients with Parkinson's disease performed several different stereotyped elbow flexion tasks, and the electromyographic (EMG) patterns from biceps and triceps were compared with previously established normal standards. The EMG pattern during a smooth flexion task was almost always abnormal and was characterised by alternating activity in biceps and triceps. The EMG patterns during a fast flexion task were also usually abnormal although they were always composed of bursts of EMG activity of normal duration appearing alternately in the agonist and antagonist muscles. These bursts, associated with movements of the limb, have a superficially similar appearance to the EMG bursts seen with tremor-at-rest, but certain physiological differences are demonstrated. This study demonstrates that both slow (ramp) and fast (ballistic) movements are clearly abnormal in these patients with disease of the basal ganglia. In a task designed to investigate antagonist inhibition before agonist activity, a majority of the patients performed normally. This suggests that, contrary to previous claims, slowness of movement (akinesia/bradykinesia) is not due either to failure to relax or to rigidity of antagonist muscles.

Abnormalities of movement which characterise patients with Parkinson's disease have traditionally been considered in terms of positive signs, such as tremor-at-rest and rigidity, or negative signs, including akinesia or bradykinesia or both. The loss of normal facility, described by the latter two terms, plus dysfunction of other as yet undefined elements of motility appear to account for more of the abnormal voluntary movements seen in this disease than do the more obvious rigidity or tremor-at-rest. Rigidity and tremor have received more investigative attention, and there have been fewer studies defining and quantitating the negative signs of Parkinson's disease. We have previously defined normal limits for the EMG correlates of certain stereotyped voluntary movements of the forearm at the elbow and described abnormalities of these movements in patients with cerebellar deficits (Hallett et al., 1975a, b). In this study, the performance during these tasks of a

\footnotetext{
${ }^{1}$ Supported by the Parkinson Disease Project of the Massachusetts General Hospital.

${ }^{2}$ Present address: Peter Bent Brigham Hospital and Harvard Medical School, Boston, Massachusetts, USA.

${ }^{3}$ Address for reprint requests: $\operatorname{Dr}$ R. R. Young, Massachusetts

General Hospital, Boston, MA 02114 USA.

Accepted 16 July 1977
}

group of patients with Parkinson's disease is compared with these normal standards to gain further insight into the pathophysiology of voluntary movements produced by this disease of the basal ganglia, particularly those movements affected by negative signs.

\section{Patients and methods}

Twenty-four patients with Parkinson's disease were studied. They were mildly (stage 1 or 2; Hoehn and Yahr, 1967) or moderately (stage 3) affected and displayed various degrees of bradykinesia, rigidity, and tremor. Some were untreated but most were taking different combinations of levodopa and anticholinergic medicines. Where there was a significant difference between the two sides, the more affected arm was studied, except for five patients in whom both arms were studied. Patients with marked tremor-at-rest could not comply with the requirements of the task and were excluded.

The experimental methods have previously been described in detail (Hallett et al., 1975a). In brief, surface EMG was recorded from biceps and triceps during stereotyped elbow flexions when the 
subject was attempting to match the step displacement of a line on an oscilloscope screen. Three different types of experiments were undertaken with each patient. They were asked to move a line under their control to match the step displacement by flexing the elbow (1) as rapidly as possible (FF, fast flexion), or (2) as smoothly as possible (SF, smooth flexion). During the third type of experiment, a $2 \mathrm{~kg}$ weight was attached to the arm which pulled in the direction of elbow flexion and required a tonic contraction in triceps while they attempted to keep their lines at the starting position before the step displacement. They were then asked to perform the fast flexion task, and the relation between cessation of EMG activity in triceps and the initiation of EMG activity in biceps was observed (AI, antagonist inhibition).

Unloading reflexes were also studied in certain patients. They contracted biceps tonically to match the starting line on the oscilloscope screen because a weight had been attached to the arm which pulled in the direction of elbow extension. Its release at any prescribed time was the stimulus for the unloading reflex. In all studies, the EMGs, the position of the experimenter's line and the subject's line, and the velocity of the subject's movement were recorded on an oscilloscope from which a permanent record was made, using a Polaroid camera.

\section{Results}

\section{FAST FLEXION}

The initial part of the EMG pattern in the FF task was entirely normal in both arms of three patients and in one arm of each of three other patients. The pattern was easily classified as 'triphasic' with bursts in biceps (B1), then in triceps (T1), then in biceps again (B2) (Fig. 1A).

One arm was studied in each of six other patients with an almost continuous tremor-at-rest; an EMG pattern of alternating activity in biceps and triceps was usually recorded preceding the triphasic pattern which moved the limb (Fig. 1B, C). B1, T1, and B2 usually occurred at approximately the same points in time that activity would have been expected to occur in biceps and triceps if the pre-existing tremor-at-rest were simply continuing. Because the voluntary movements were frequently of unusually low velocity and, therefore, slow to reach completion, it was often difficult to tell which bursts were responsible for accelerating the limb (Fig. 1C).

In 17 arms of 15 patients, although tremor-atrest was not continuously present, a single 'beat of tremor' occasionally occurred before the pattern which moved the limb (Fig. 1D, E). This beat of tremor consisted of a burst of activity in biceps (defined as B0 because it did not move the limb) followed by a silent period in that muscle (B0-B1), during which triceps activity occurred (T0); less frequently, there would be only a T0. There was no apparent difference between this isolated tremor beat and the terminal part of a continuous tremor before the movement pattern.

Quantitatively, the durations of B1, T1, and the interval between the end of $\mathrm{B} 1$ and the beginning of B2 (B1-B2) were always either well within the normal range or no more than $\pm 10 \mathrm{~ms}$ from the extremes of the normal range. The durations of the parameters $\mathrm{B} 0, \mathrm{~T} 0$, and $\mathrm{B} 0-\mathrm{B} 1$ were only slightly less than normal (Table).

Table Ranges of average durations (in $\mathrm{ms}$ ) of certain EMG parameters during stereotyped elbow flexion movements

\begin{tabular}{llll}
\hline & $B I$ & $T I$ & $B I-B 2$ \\
\hline Normal subjects & $60-105$ & $30-85$ & $40-150$ \\
Patients with Parkinson's disease & $50-100$ & $40-95$ & $40-140$ \\
$\begin{array}{l}\text { Patients with Parkinson's disease who } \\
\begin{array}{l}\text { had one or more bursts of tremor } \\
\text { before movement }\end{array}\end{array}$ & $B 0$ & $T 0$ & $B 0-B I$ \\
\hline
\end{tabular}

In normal subjects, the peak velocity of forearm movement during rapid flexion at the elbow occurs about the time of T1. Many records in the group of patients with Parkinson's disease show the peak velocity to be near the time of a second burst of activity in triceps (Fig. 1F). This could be interpreted as a ' $\mathrm{B} 1, \mathrm{~T} 1, \mathrm{~B} 2, \mathrm{~T} 2, \mathrm{~B} 3$ ' pattern or as a 'B0, T0, B1, T1, B2' pattern, with the tremor beat beginning the acceleration and $T 1$ retaining its usual relationship to the peak velocity. Regardless, the increase in velocity (acceleration) of elbow flexion is clearly slower in these patients than in the normal subjects.

Whereas B1 (Garland and Angel, 1971) and T1 (Hallett et al., 1975a) are relatively unaffected, B2 and later components are significantly affected by perturbations of the input from the peripheral reflex servoloop such as are responsible for an unloading reflex. Because EMG patterns during tremor-at-rest (Shahani and Young, 1976) appear similar to the patterns seen during ballistic movements with respect to both the alternation of EMG activity in agonist and antagonist muscles and the duration of the EMG bursts, tremor-at-rest was studied in three patients using the unloading reflex. The biceps component of this tremor, includ- 


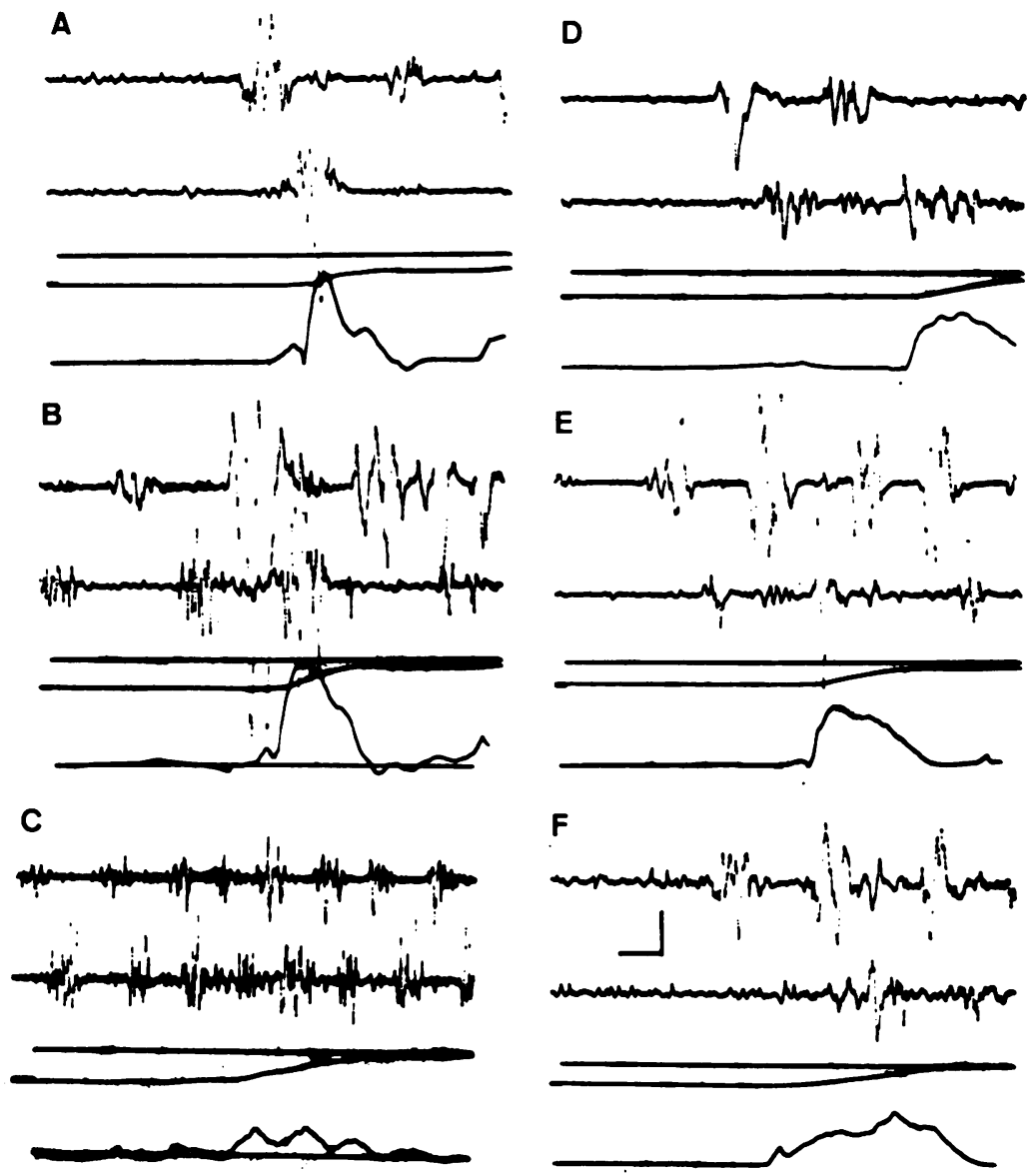

Fig. 1 Fast flexion patterns in patients with Parkinson's disease. In each part of the figure, the traces represent from above downward: biceps EMG, triceps EMG, the step to be matched, the position of the patient's arm and the velocity of the arm. See text for description of individual records.

Calibration (parts $A, B, D-F): 50 \mathrm{~ms}, 200 \mu \mathrm{V} ;($ part C): $100 \mathrm{~ms}, 200 \mu \mathrm{V}$.

ing the initial part of the tremor burst, proved to be sensitive to unloading - that is, a silent period was present in response to unloading and could significantly diminish expected biceps activity (Fig. 2).

\section{SMOOTH FLEXION}

Electromyographic patterns from normal subjects during the SF task are characterised by continuous or, less frequently, intermittent activity in biceps with little activity in triceps-the latter appearing only simultaneously with biceps, never by itself. A normal pattern was always seen in three affected arms of three patients (Fig. 3A). More commonly in patients with Parkinson's disease (seen at least occasionally in 23 arms of 21 patients), an alternating pattern of activity in biceps and triceps was seen during these slower flexion movements (Fig. 3B, C). This alternating pattern is usually rather irregular, but the feature that differentiates it from normal is the presence of triceps EMG activity at a time when biceps is relatively silent. The actual movement itself can be quite smooth despite the irregular, alternating nature of the EMG. In a few patients with prominent tremor-atrest, this alternating pattern persisted during the initial part of the movement. (Fig. 3D).

ANTAGONIST INHIBITION

In the AI task, EMG activity in triceps normally 


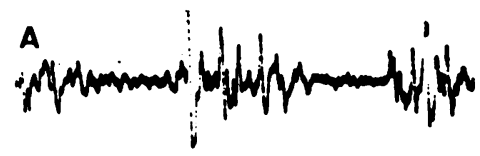

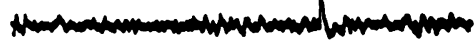

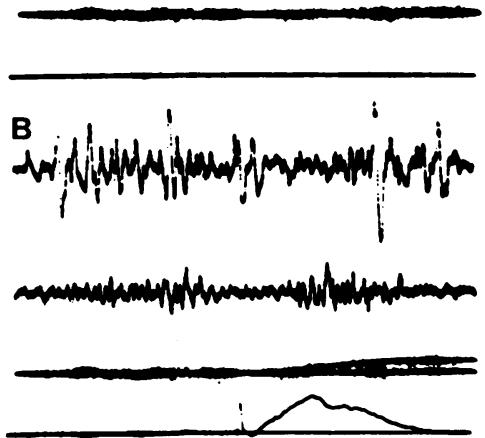

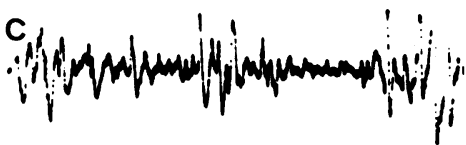

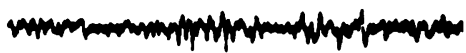
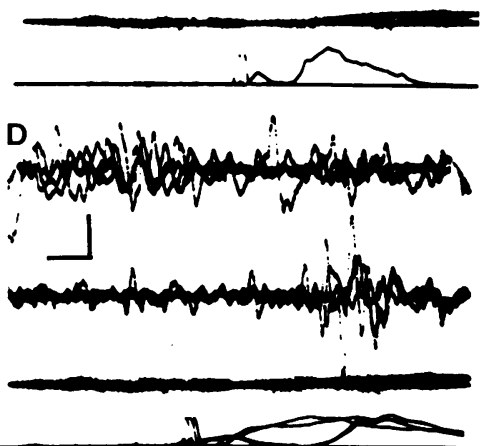

Fig. 2 Unloading reflex in a patient with Parkinson's disease with prominent resting tremor. Patient's arm is connected to a $750 \mathrm{gm}$ weight pulling in the direction of elbow extension. Traces are the same as in Fig. I with the addition of a sixth trace which indicates by a vertical mark the time of rclease of the electromagnet holding the weight. A shows the pattern without release. $B$ and $C$ are examples of single traces with weight release, and decrease of expected biceps activity can be appreciated. $D$ is three superimposed traces with unloading and shows the silent period in biceps. Calibration (parts $A-C$ ): $50 \mathrm{~ms}, 100 \mu \mathrm{V}$; (part D): $20 \mathrm{~ms}, 100 \mu \mathrm{V}$.

A
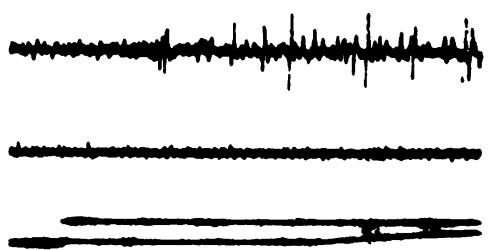

B

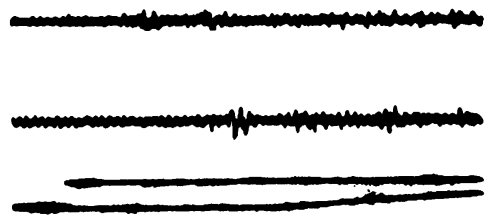

C
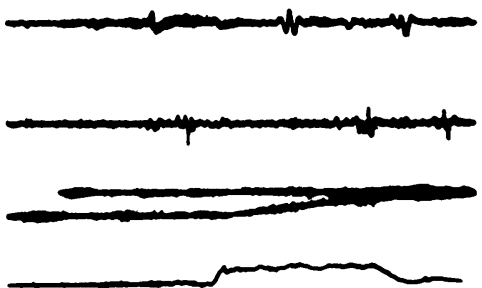

D

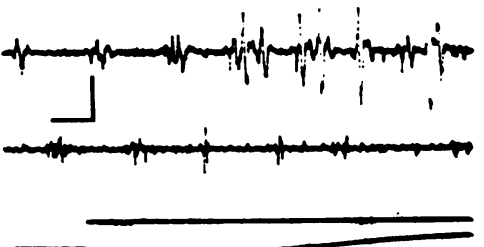

Fig. 3 Smooth flexion patterns in patients with Parkinson's diseare. Traces are the same as in Fig. 1. See text for description of individual records. Calibration (parts A, B) $100 \mathrm{~ms}, 100 \mu \mathrm{V}$; (parts C, D) $100 \mathrm{~ms}, 200 \mu \mathrm{V}$. 
ceases sometime during the $50 \mathrm{~ms}$ before the start of EMG activity in biceps. This behaviour was seen in 20 arms of 17 patients. Less frequently (in six arms of five patients), there was occasionally some overlap in the activity of the two muscles.

There was no obvious correlation between the presence or degree of abnormality recorded during these tests and the severity of any of the signs of Parkinson's disease or results of therapy. More definitive studies, however, remain to be done.

\section{Discussion}

A prominent feature of voluntary movements of patients with Parkinson's disease is bradykinesia, or slowness of movement (Draper and Johns, 1964; Flowers, 1975, 1976). This term, as used clinically, includes prolongation of reaction time, as well as decreased velocity and acceleration once the movement is begun. Because this study has shown the duration of EMG bursts during attempted fast flexion activity and the relation of EMG activity in agonist and antagonist muscles to be normal, prolongation of the former or cocontraction of the antagonist muscle cannot account for bradykinesia. Activity of agonist and antagonist in our patients' rapid movements was, for the most part, alternating, as is the case normally. This is in contrast to the abnormal cocontraction seen during exteroceptive polysynaptic reflexes (Delwaide et al., 1974) in patients with Parkinson's disease. A partial explanation for bradykinesia may include abnormalities of the patterns described above. Some of the delay in initiating movement in patients with tremor-at-rest might come from 'waiting to get into the correct time of the cycle' for a spontaneous burst in biceps in order to initiate the movement, using that activity as B1. In patients without apparent tremor-at-rest, a similar delay might come from having to produce 'a beat of tremor' before the movement. Some of the decrease in velocity of the movements is reflected in the fact that many movements in the patients with Parkinson's disease were not completed during a single cycle of biceps and triceps activity but appeared to require acceleration over two cycles (Fig. 1F). Perhaps the amount of EMG activity in the initial biceps and triceps bursts cannot be increased sufficiently to produce a long rapid movement and, thus, a combination of several sets of EMG bursts are needed. This may correspond to Flowers's observation in patients with Parkinson's disease, that short initial movements and corrective movements were accomplished in a normal amount of time, but that larger amplitude movements were slow to be completed (Flowers, 1976).

The apparent electromyographic similarity between rapid voluntary alternating movements and tremor-at-rest in patients with Parkinson's disease has been noted for many years (Hoefer and Putnam, 1940; Bishop et al., 1948). It has recently been shown that afferent activity from human muscle spindles during these two movements is identical (Hagbarth et al., 1975). A tremor can be constructed artificially from the normal fast flexion movement pattern which would then have at least a superficial similarity to voluntary alternating movements or tremor-at-rest. Let us assume that an average normal duration of B1 of $80 \mathrm{~ms}$ and of B1-B2 of $95 \mathrm{~ms}$ (Hallett et al., 1975a) produces a total cycle time of $175 \mathrm{~ms}$. If a new cycle of B1 and B1-B2 were begun when a B1-B2 interval was completed and triceps activity alternated with biceps activity, a $5 \frac{1}{2}$ to $6 \mathrm{~Hz}$ 'tremor' would be produced. Perhaps the tremorat-rest in patients with Parkinson's disease is, to paraphrase Alberts (1972), the involuntary and repetitive running of the programme for single voluntary rapid movements. On the other hand, there are certain differences between this artificially constructed tremor and the tremor-at-rest. The duration and amplitude of B1 are relatively resistant to modification by the unloading reflex (Garland and Angel, 1971), whereas the biceps component of tremor-at-rest was rather sensitive to unloading. The durations of the tremor bursts (at least as exemplified by those bursts directly preceding the voluntary movement, $\mathrm{B} 0$ and $\mathrm{T} 0$ ) are slightly shorter on average than the bursts with fast flexion (B1 and T1). Although the tremor-at-rest in patients with Parkinson's disease can reach $6 \mathrm{~Hz}$, more typically it has a frequency in the range of 4-5 Hz. Finally, the tremor-atrest is diminished or eliminated by voluntary movements of the sort needed to produce the triphasic EMG pattern.

Why the fast flexion pattern of a rapid movement usually fits the cycle of a previously present tremor-at-rest is uncertain, but by analogy with engineering models of efficient movement (Greene, 1972), there may be value in having a low level oscillation of the part to be moved before the initiation of a major movement. Previous studies (Petajan and Jarcho, 1975) have shown that, when electrophysiological recordings are made, tremor is a more frequent abnormality in Parkinson's disease than is suspected clinically. In our studies, tremor intrudes itself into the movements even when it is not present electrophysiologically at rest. In attempted fast movements, a single 'beat 
of tremor' may appear before the movement. A fast flexion effort might require several cycles of the triphasic pattern which would then resemble resting tremor. Attempted smooth flexion movements are characterised by alternating activity in flexors and extensors, and the observations of Petajan and Jarcho that single motor units tend to be recruited at the tremor frequency in Parkinson's disease may help account for this.

This inability to generate a normal EMG pattern in the smooth flexion task is another difficulty identified here for patients with Parkinson's disease. Even when the movement is clinically or mechanically smooth, the EMG pattern is often broken up with alternating EMG activity in agonist and antagonist muscles. Although the clinical significance of this fact is unclear, the result agrees with several previous lines of investigation. Grimby and Hannerz (1974) have shown that movements of patients with Parkinson's disease often begin with an unstable recruitment order of single motor units, which they interpret as being a phasic pattern. Petajan and Jarcho (1975) show that single motor units in these patients who attempt tonic movements often fire in bursts reminiscent of tremor rather than in a normal asynchronous pattern.

Kornhuber $(1971,1974)$ suggested that lesions of the basal ganglia would affect 'ramp generation' for slow, voluntary movements of different speeds. DeLong and Strick (1974) demonstrated in the monkey that large neurones in the striatum are more active during smooth, ramp movements than during fast, ballistic movements. Because patients with Parkinson's disease, have, as a primary abnormality, decreased nigrostriatal input, it might be expected that their smooth movement (ramp) patterns would be more disturbed than their fast flexion (ballistic) patterns. In fact, both are shown to be abnormal. On the one hand, alternating tremulous activity during movement (separate from tremor-at-rest) complicates both slow and fast movements. More important, the slow ramp movements are otherwise relatively undisturbed, whereas the fast movements are very much slower than normal. This is in keeping with the clinician's impression that patients with Parkinson's disease can usually perform fairly complex movements and reach normal peak power, but both types of activity are done very slowly-that is, ordinarily rapid movements are performed slowly, whereas slow movements are not as obviously affected. These clinical facts appear to be at variance with Kornhuber's predictions.

Kornhuber also states that 'akinesia in Parkinson syndrome, however, consists of a defect in ramp generation' and ' . . . in Parkinson akinesia some ramp movements are performed very slowly ... '. The only defect in ramp movements noted in this study was action tremor very different from akinesia. Akinesia and bradykinesia are clinical terms used to describe a complex abnormality (or abnormalities) of the initiation or regulation of movement. These concepts are not easily or with certainty translated into quantifiable abnormalities of simple behaviour such as flexion of the elbow. It is not possible now for us to provide a method for quantifying akinesia with which everyone will agree, though the technique used here can measure the speed, or lack of it, in performance of various stereotyped elbow flexion tasks.

Despite their bradykinesia and the abnormalities of EMG patterns found during fast flexion, all patients were, at one time or another (but not repeatedly), able to make relatively rapid movements. The surprising variability of the patients' responses so that they could do this occasionally might reflect the unusual situation of the experimental laboratory. A clinical analogy would be 'paradoxical kinesia' (Schwab, 1972). Althoughọ $\vec{N}$ patients with Parkinson's disease might, during exceptional circumstances, be able to generateo $\vec{N}$ triphasic patterns and rapid movements, their ${ }_{\overparen{O}}$ disease would ordinarily prevent them from using this sort of motor programme.

Results in the AI task indicate that, for patients with Parkinson's disease, antagonist inhibition is usually related appropriately to the initiation of activity in the agonist. In occasionalo patients, however, there is a slight overlap of activity in the two muscles. This abnormality is rather frequent, and often much more severe, in patients with cerebellar deficits where it was thought possibly to be related to dysdiadochokinesis (Hallett et al., 1975b). There is quite often in Parkinson's disease a clumsiness in alternating movements that is not very well defined and not clearly separable from clumsiness seen in other movement disorders (Fisher, 1960, 1961; Schwab, 1972); this abnormality in antagonist inhibition may occasionally underlie some aspects of 'clumsiness'. The results of this experiment dispute the claim that slowness of movement in Parkinson's disease is due to 'failure to relax' or rigidity of the antagonist muscles.

\section{References}

Alberts, W. W. (1972). A simple view of Parkinsonian tremor. Electrical stimulation of cortex adjacent to the Rolandic fissure in awake man. Brain Research, 44, 357-369. 
Bishop, G. H., Clare, M. H., and Price, J. (1948). Patterns of tremor in normal and pathological conditions. Journal of Applied Physiology, 1, 123147.

DeLong, M. E., and Strick, P. L. (1974). Relation of basal ganglia, cerebellum and motor cortex units to ramp and ballistic limb movements. Brain Research, 71, 327-335.

Delwaide, P. J., Schwab, R. S., and Young, R. R. (1974). Polysynaptic spinal reflexes in Parkinson's Disease. Neurology (Minneapolis), 24, 820-827.

Draper, I. T., and Johns, R. J. (1964). The disordered movement of Parkinsonism and the effect of drug treatment. Bulletin of the Johns Hopkins Hospital, 115, 465-480.

Fisher, C. M. (1960). A simple test of coordination in the fingers. Neurology (Minneapolis), 10, 745-746.

Fisher, C. M. (1961). An improved test of motor coordination in the lower limbs. Neurology (Minneapolis), 11, 335-336.

Flowers, K. A. (1975). Ballistic and corrective movements on an aiming task: intention tremor and Parkinsonian movement disorders compared. Neurology (Minneapolis), 25, 413-421.

Flowers, K. A. (1976). Visual 'closed-loop' and 'openloop' characteristics of voluntary movement in patients with Parkinsonism and intention tremor. Brain, 99, 269-310.

Garland, H., and Angel, R. W. (1971). Spinal and supraspinal factors in voluntary movement. Experimental Neurology, 33, 343-350.

Greene, P. H. (1972). Problems of organization of motor systems. In Progress in Theoretical Biology. Vol. 2. pp. 303-338. Edited by R. Rose and F. M. Snell. Academic Press: New York.

Grimby, L., and Hannerz, J. (1974). Disturbances in the voluntary recruitment order of anterior tibial motor units in bradykinesia of Parkinsonism. Journal of Neurology, Neurosurgery, and Psychiatry, 37, 47-54.

Hagbarth, K. E.. Wallin. G.. Löfstedt, L., and Aquilonius, S. M. (1975). Muscle spinclle activity in alternating tremor of Parkinsonism and in clonus. Journal of Neurology, Neurosurgery, and Psychiatry, 38, 636-641.

Hallett, M., Shahani, B. T., and Young, R. R. (1975a). EMG analysis of stereotyped voluntary movements in man. Journal of Neurology, Neurosurgery, and Psychiatry, 38, 1154-1162.

Hallett, M., Shahani, B. T., and Young, R. R. (1975b). EMG analysis of patients with cerebellar deficits. Journal of Neurology, Neurosurgery, and Psychiatry, 38, 1163-1169.

Hoefer, P. F. A., and Putnam, T. J. (1940). Action potentials of muscles in rigidity and tremor. Archives of Neurology and Psychiatry (Chicago), 43, 704-725.

Hoehn, M. M., and Yahr, M. D. (1967). Parkinsonism: onset, progression and mortality. Neurology (Minneapolis), 17, 427-442.

Kornhuber, H. H. (1971). Motor functions of cerebellum and basal ganglia: the cerebello-cortical saccadic (ballistic) clock, the cerebello-nuclear hold regulator, and the basal ganglia ramp (voluntary speed smooth movement) generator. Kybernetik, 8, 157-162.

Kornhuber, H. H. (1974). Cerebral cortex, cerebellum, and basal ganglia: an introduction to their motor functions. In The Neurosciences Third Study Program, pp. 267-280. Edited by F. O. Schmitt and F. G. Worden. MIT Press: Cambridge, Massachusetts.

Petajan, J. H., and Jarcho, L. W. (1975). Motor unit control in Parkinson's Disease and the influence of levodopa. Neurology (Minneapolis), 25, 866-869.

Schwab, R. S. (1972). Kinesia paradoxica. In Recent Contributions to Neurophysiology, pp. 87-92. Edited by J. P. Cordeau and P. Gloor. Elsevier Publishing Company: Amsterdam.

Shahani, B. T., and Young, R. R. (1976). Physiological and pharmacological aids in the differential diagnosis of tremor. Journal of Neurology, Neurosurgery, and Psychiatry, 39, 772-783. 